\title{
Leader Selection Problem for Stochastically Forced Consensus Networks based on Matrix Differentiation
}

\author{
Leitao Gao ${ }^{\mathrm{a}}$, Guangshe Zhao ${ }^{\mathrm{a}}$, Guoqi $L i^{* \mathrm{~b}}$, Zhaoxu Yang ${ }^{\mathrm{c}}$, \\ ${ }^{a}$ School of Electronic and Information Engineering, Xi'an Jiaotong University, Xi'an, \\ Shaanxi, P.R. China,710049. \\ ${ }^{b}$ Center for Brain Inspired Computing Research, Department of Precision Instrument, \\ Tsinghua University, Beijing, P.R. China,100084. \\ ${ }^{c}$ School of Aerospace, Xi'an Jiaotong University, Xi'an, Shaanxi, P.R. China,710049.
}

\begin{abstract}
The leader selection problem refers to determining a predefined number of agents as leaders in order to minimize the mean-square deviation from consensus in stochastically forced networks. The original leader selection problem is formulated as a non-convex optimization problem where matrix variables are involved. By relaxing the constraints, a convex optimization model can be obtained. By introducing a chain rule of matrix differentiation, we can obtain the gradient of the cost function which consists matrix variables. We develop a "revisited projected gradient method" (RPGM) and a "probabilistic projected gradient method" (PPGM) to solve the two formulated convex and non-convex optimization problems, respectively. The convergence property of both methods is established. For convex optimization model, the global optimal solution can be achieved by RPGM, while for the original non-convex optimization model, a suboptimal solution is achieved by PPGM. Simulation results ranging from the synthetic to real-life networks are provided to show the effectiveness of RPGM and PPGM. This works will deepen the understanding of leader selection problems and enable applications in various real-life distributed control problems.
\end{abstract}

Keywords: Leader selection, Consensus, Stochastically forced networks, Revisited projected gradient method (RPGM), Probabilistic projected gradient method (PPGM)

Email address: liguoqi@mail.tsinghua.edu.cn. (Guoqi Li*)

Preprint submitted to Elsevier

November 23, 2016

(C) 2016. This manuscript version is made available under the Elsevier user license

http://www.elsevier.com/open-access/userlicense/1.0/ 


\section{Introduction}

Reaching consensus in multi-agent networks has gained much attention in recent years, particularly in distributed systems and social networks [1-4]. Agents in these systems are able to agree on a common value of interests via a local interaction protocol. For example, in social networks, a group of individuals are used to agree on a certain value $[5,6]$. Recently, consensus problems have received considerable attention in the context of distributed control, including the control of unmanned vehicle formations and coordination of mobile autonomous agents $[7,8]$. When agents in these systems are trying to agree on a certain value, they must maintain this agreement in the face of external disturbances and unreliable communications [9-11]. Thus, it is important to study the robustness of consensus in the networks where all nodes are subject to stochastic disturbances ( termed as stochastically forced networks) [12-15]. To evaluate a consensus protocol in stochastically forced networks, the communication graph and the mean-square deviation from consensus should be utilized [16-21].

In this paper, we consider undirected consensus networks with two groups of nodes subject to stochastic disturbances. This type of networks are generally formulated as leader-follow systems. In a leader-follower system, the leaders act as external inputs to steer the whole group, and the followers update their states based on the information available from their neighbors. For example, in unmanned vehicle formations control [7], a vehicle is a leader if it has GPS devices, and other vehicles are followers. The local interactions among these vehicles can be used to reach an agreement on heading angle, velocity, inter-vehicular spacing, etc. Thus, to select the vehicle with GPS which can steer the rest of vehicles more effectively becomes significant. This is often referred as the leader selection problem. In real-life applications, the leaders may be in the face of external disturbances, which may prevent it from interacting with followers following the desired trajectories. Therefore, it is important to consider leader selection problem in stochastically forced consensus networks.

Leader selection problem has been extensively studied in recent years [2128]. Leaders were traditionally selected based on majority voting or degreebased approaches [22]. [23] showed that leader selection problem could be relaxed to a semidefinite program and solved efficiently by greedy algorithms 
(GA). [24] proposed an information-centrality-based formulation that a node in the network graph with maximal information centrality became a leader. The notion of manipulability index in [26] was used to measure the influence of leaders' inputs on the network centroid to select leaders. It has been proven that optimal leader selection in linear multi-agent systems with noisy links was a sub-modular optimization problem [25], which could be solved by minimizing the total error covariance. Then, the lower bound could be obtained by minimizing the mean-square deviation. By using greedy algorithm (GA) to identify leaders, a customized algorithm was developed for large networks [21]. A graph-theoretic approach in [27] showed that an upper bound and a lower bound of the convergence rate to the consensus value were derived based on the maximum distance from leaders to followers. [28] proposed a projected gradient method (PGM) to minimize the cost control and used PGM-extension (PGME) to select key nodes in directed networks.

In this work, we are interested in assigning a predefined number of nodes as leaders in order to minimize the mean-square deviation from consensus in stochastically forced networks. Specifically, we formulate the original leader selection problem as a non-convex optimization model where matrix variables are involved. By relaxing the constraints to a hyperplane, a convex optimization model can also be obtained. Therefore, a key procedure is to obtain the gradient of the cost function. However, it is difficult to take the derivative of a function with respect to a matrix variable which may be depending on other intermediate matrix variables. To this end, we introduce a chain rule [28] to address the issue of matrix differentiation. A projected gradient method (PGM) [28, 29] is revisited, namely, revisited PGM (RPGM), to solve the convex optimization problem and the global optimal solution (lower bound) can be obtained. In contrast to greedy algorithm (GA) and PGM-extension (PGME) [28], we develop a probabilistic projected gradient method (PPGM) to select the leaders. We have also applied RPGM, PPGM, GA and PGME to synthetic and real-life networks. Simulation results show that i) PPGM is simpler than GA but with comparable performance; ii) PPGM steadily approaches RPGM and outperforms PGME.

This paper is organized as follows. In Section II, we formulate the leader selection problem and build two models, one is the original non-convex optimization model and the other is a convex optimization model by relaxing the constraints on a hyperplane. In Section III, we develop the revisited projected gradient method (RPGM) and probabilistic projected gradient method (PPGM) to solve the two proposed optimization problems, respectively. In 
Section IV, the convergence property of both methods is established. Section $\mathrm{V}$ contains simulations results in both synthetic and real-life networks. We summary this work in section VI.

\section{Problem Formulation}

The control objective is to strategically deploy leaders in order to reduce the variance amplification in stochastically forced consensus networks. In this section, we consider a connected, undirected network of $N$ identical agents, with a time-invariant communication structure. The network is modelled by an undirected graph $G=(V, E)$, where $V$ is the set of nodes (with $|V|=N$ ) and $\mathrm{E}$ is the set of edges (with $|E|=m$ ). The Laplacian matrix [30] of $G$ is

$$
L=\widehat{D}-A
$$

where $\widehat{D}$ is the diagonal matrix of node degrees and $A$ is the adjacency matrix of $G$. The Laplacian $L$ is a positive semidefinite matrix.

In an undirected network, the state of the system is given $\varphi=\left[\varphi_{1}, \varphi_{2}, \ldots, \varphi_{N}\right]$, where $\varphi_{i}$ is the state of agent $i$. Each node updates a scalar state $\varphi$ as follows

$$
\dot{\varphi}=u_{i}+w_{i}
$$

where $u_{i}$ is the control law and $w_{i}$ is the white stochastic disturbance with

zero-mean and unit-variance. A node is a follower if it uses only relative information exchange with its neighbors to form its control action

$$
u_{i}=-\sum_{j \in\left(N_{i}\right)}\left(\varphi_{i}-\varphi_{j}\right)
$$

where $N_{i}$ is the set of neighbors of node $i$. A node is a leader if, in addition to relative information exchange with its neighbors, it also has access to its own state

$$
u_{i}=-\sum_{j \in\left(N_{i}\right)}\left(\varphi_{i}-\varphi_{j}\right)-k_{i} \varphi_{i}
$$

where $k_{i}$ is a pre-given positive parameter.

A state-space representation of leader-follower consensus network is given by

$$
\dot{\varphi}=-(L+D X) \varphi+w
$$


where $w$ is the white stochastic disturbance with zero-mean and unit-variance, and

$$
D:=\operatorname{diag}(k) ; \quad X:=\operatorname{diag}(x)
$$

are diagonal matrix formed from the vectors $k=\left[k_{1} \ldots k_{N}\right]^{\prime}$ and $x=\left[x_{1} \ldots x_{N}\right]^{\prime}$. Here, $x$ is a Boolean-valued vector with its $i$ th entry $x_{i} \in\{0,1\}$, indicating that node $i$ is a leader if $x_{i}=1$ and a follower if $x_{i}=0$.

In this work, the objective is to minimize the steady-state variance of the network given by:

$$
\varepsilon_{s s}=\lim _{t \rightarrow \infty} \varepsilon\left(\varphi(t) \varphi^{\mathrm{T}}(t)\right)
$$

where $\varepsilon\left(w(t) w^{\mathrm{T}}(t)\right)=I \delta(t-\tau), \varepsilon(\cdot)$ is the expectation operator [21]

Lemma 1. $\varepsilon_{s s}$ is the limit value of $\varepsilon(\cdot)$, where $\varepsilon(\cdot)$ is the expectation operator of the $\varphi$. Then the steady-state variance $\varepsilon_{s s}$ is the solution to the Lyapunov equation

$$
(L+D X) \frac{\varepsilon_{s s}}{2}+\frac{\varepsilon_{s s}}{2}(L+D X)^{\mathrm{T}}=I
$$

Proof. Noting that $H=\varepsilon\left[\varphi(t) \varphi^{\mathrm{T}}(t)\right]$, we have

$$
\begin{aligned}
\dot{H} & =\varepsilon\left[\dot{\varphi}(t) \varphi^{\mathrm{T}}(t)+\varphi(t) \dot{\varphi}^{\mathrm{T}}(t)\right] \\
& =\varepsilon\left\{[-(L+D X) \varphi(t)+w] \varphi^{\mathrm{T}}(t)+\varphi(t)\left[\varphi^{\mathrm{T}}(t)(-(L+D X))^{\mathrm{T}}+w^{\mathrm{T}}\right]\right\} \\
& =\varepsilon\left\{\left[-(L+D X) \varphi(t) \varphi^{\mathrm{T}}(t)+w \varphi^{\mathrm{T}}(t)\right]+\left[\varphi(t) \varphi^{\mathrm{T}}(t)(-(L+D X))^{\mathrm{T}}\right.\right. \\
& \left.\left.+\varphi(t) w^{\mathrm{T}}\right]\right\} \\
& =-(L+D X) \varepsilon\left[\varphi(t) \varphi^{\mathrm{T}}(t)\right]+\varepsilon\left[w \varphi^{\mathrm{T}}(t)\right]+\varepsilon\left[\varphi(t) \varphi^{\mathrm{T}}(t)\right](-(L+D X))^{\mathrm{T}} \\
& +\varepsilon\left[\varphi(t) w^{\mathrm{T}}\right]
\end{aligned}
$$

Thus, we obtain that

$$
\dot{H}=-(L+D X) H-H(L+D X)^{\mathrm{T}}+\varepsilon\left[w \varphi^{\mathrm{T}}(t)\right]+\varepsilon\left[\varphi(t) w^{\mathrm{T}}\right]
$$

It is known that $\varphi(t)=e^{-(L+D X)(t)} \varphi(0)+\int_{0}^{t} e^{-(L+D X)(t-\tau)} w(\tau) d \tau$, and as $w$ is the white stochastic disturbance, we obtain that

$$
\begin{aligned}
& \varepsilon\left[\varphi(t) w^{\mathrm{T}}\right] \\
& =\varepsilon\left[e^{-(L+D X)(t)} \varphi(0) w^{\mathrm{T}}+\int_{0}^{t} e^{-(L+D X)(t-\tau)} w(\tau) w^{\mathrm{T}} d \tau\right] \\
& =e^{-(L+D X)(t)} \varepsilon\left[\varphi(0) w^{\mathrm{T}}\right]+\int_{0}^{t} e^{-(L+D X)(t-\tau)} \varepsilon\left[w(\tau) w^{\mathrm{T}}\right] d \tau \\
& =\int_{0}^{t} e^{-(L+D X)(t-\tau)} \varepsilon\left[w(\tau) w^{\mathrm{T}}\right] d \tau \\
& =I
\end{aligned}
$$


Combining (2) and (9), we have

$$
\begin{aligned}
\dot{H} & =-(L+D X) H-H(L+D X)^{\mathrm{T}}+I+I \\
& =-(L+D X) H-H(L+D X)^{\mathrm{T}}+2 I
\end{aligned}
$$

Note that $L+D X$ is a symmetric positive definite matrix as long as there is at least one $(D X)_{i i}>0$ for $i=1, \ldots, N[31]$. Therefore in steady state we have $\lim _{t \rightarrow \infty} \dot{H}=0$. We finally obtain that

$$
(L+D X) \frac{\varepsilon_{s s}}{2}+\frac{\varepsilon_{s s}}{2}(L+D X)^{\mathrm{T}}=I
$$

This lemma holds

The solution for the equation (7) is obtained by $\varepsilon_{s s}=(L+D X)^{-1}[14,16]$. Thus, we use the total steady-state variance

$$
\operatorname{trace}\left(\varepsilon_{s s}\right)=\operatorname{trace}\left[(L+D X)^{-1}\right]
$$

as an cost function to quantity the performance of stochastically forced consensus networks.

Recall that the aim is to identify $N_{l}$ leaders that are most effective in reducing the steady-state variance. For a predefined number of leaders $N_{l}<$ $N$, the leader selection problem can be formulated as:

$$
\begin{array}{ll}
\text { minimize } & J(X)=\operatorname{trace}\left((L+D X)^{-1}\right) \\
\text { subject to } & x_{i} \in\{0,1\}, \quad i=1, \ldots, N \\
& \mathbf{1}^{\mathrm{T}} x=N_{l}
\end{array}
$$

Here the Laplacian matrix $L$ as well as diagonal matrix $D$ consisting all $k_{i}$ are pre-given, and the matrix $X$ is the variable to be determined. Due to the Boolean constraints of $x_{i} \in\{0,1\}$, the model formulated in (12) is a non-convex optimization model. By removing the Boolean constraints, the following convex optimization model can be formulated:

$$
\begin{aligned}
\text { minimize } & J(X) & =\operatorname{trace}\left((L+D X)^{-1}\right) \\
\text { subject to } & \mathbf{1}^{\mathrm{T}} x & =N_{l}
\end{aligned}
$$

where $X \in R^{N \times N}$ and $\mathbf{1}^{\mathrm{T}} x=N_{l}$ denotes the relaxed hyperplane.

In order to solve the model (12) and (13), we define a normal function $N(X)=\operatorname{tr}(X)$ to associate with the equality constraint on $X$. Two major contributions will be shown in later sections: 1) we develop the RPGM to 
solve convex optimization model (13) and the global optimal solution can be obtained asymptotically; 2) we propose PPGM to solve the non-convex optimization model (12) and a suboptimal solution can be achieved in a few iterations.

\section{3. problem solution with a new algorithm}

In order to obtain the gradient of the cost function, some lemmas are introduced first.

Lemma 2. [28] Chain rule: Suppose that matrix $U \in R^{N \times M}$ is a function of matrix $X$, i.e., $U=g(X)$, the derivative of the function $E(U)=E(g(X))$ with respect to $X$ is given by the chain rule as follows:

$$
\begin{aligned}
\frac{\partial E(g(X))}{\partial X_{i j}} & =\sum_{m, n} \frac{\partial E(U)}{\partial U_{m n}} \frac{\partial U_{m n}}{\partial X_{i j}} \\
& =\sum_{m, n}\left[\frac{\partial E(U)}{\partial U}\right]_{m n}\left[\frac{\partial U}{\partial X_{i j}}\right]_{m n} \\
& =\sum_{m, n}\left[\frac{\partial E(U)}{\partial U}\right]_{n m}^{\mathrm{T}}\left[\frac{\partial U}{\partial X_{i j}}\right]_{m n} \\
& =\sum_{n}\left(\left[\frac{\partial E(U)}{\partial U}\right]^{\mathrm{T}}\left[\frac{\partial U}{\partial X_{i j}}\right]\right)_{n n}
\end{aligned}
$$

As it is known that $\operatorname{tr}(A)=\sum_{i}(A)_{i i}$, we have

$$
\begin{aligned}
\frac{\partial E(g(X))}{\partial X_{i j}} & =\sum_{n}\left(\left[\frac{\partial E(U)}{\partial U}\right]^{\mathrm{T}}\left[\frac{\partial U}{\partial X_{i j}}\right]\right)_{n n} \\
& =\operatorname{tr}\left(\left[\frac{\partial E(U)}{\partial U}\right]^{T}\left[\frac{\partial U}{\partial X_{i j}}\right]\right) \\
& =\frac{\partial E(U)}{\partial U_{m n}} \cdot \frac{\partial U_{m n}}{\partial X_{i j}}
\end{aligned}
$$

where $X_{i j}$ is the $i j$-th element of matrix $X$, and the indexes $\sum_{m, n}(1 \leq$ $m \leq M, 1 \leq n \leq N)$ is omitted as the summation over repeated indices is implied (usually referred to as Index-notation-arrangement or Einstein notation). Thus, the $i j$-th element of matrix $\frac{\partial E(g(X))}{\partial X}$, which is the derivative of $E(g(X))$ with respect to matrix $X$, denoted as $\left[\frac{\partial E(g(X))}{\partial X}\right]_{i j}$ is obtained by

$$
\left[\frac{\partial E(g(X))}{\partial X}\right]_{i j}=\frac{\partial E(g(X))}{\partial X_{i j}}
$$


Lemma 3. [32] For a matrix $X$ with no special structure(i.e., elements of $X$ are independent), denote $[X]_{i j}$ as the $i j$-th element of matrix $X$ and $\delta_{i j}=1$ if $i=j$ (otherwise, $\delta_{i j}=0$ ). We have:

1) $\frac{\partial X_{k l}}{\partial X_{i j}}=\delta_{k i} \cdot \delta_{l j}$

2) $\frac{\partial X^{-1}}{\partial X}=-X^{-T} X^{-T}$

3) For matrix $A, B, X$ such that $[X]_{i j}=\sum_{k}[A]_{i k}[B]_{k j}$ (writen as $[X]_{i j}=$ $\left.[A]_{i k} \cdot[B]_{k j}\right)$, then $X=A B$.

Lemma 4. The gradient of the function $J(X)$ is

$$
\frac{\partial J(X)}{\partial X}=-D^{\mathrm{T}}(L+D X)^{-\mathrm{T}}(L+D X)^{-\mathrm{T}}
$$

Proof. From the Lemma 2 and Lemma 3, we have

$$
\begin{aligned}
& \frac{\partial J(X)}{\partial X_{i i}} \\
& =\frac{\partial \operatorname{tr}\left((L+D X)^{-1}\right)}{\partial X_{i i}} \\
& =\frac{\partial \operatorname{tr}\left((L+D X)^{-1}\right)}{\partial(L+D X)_{p q}} \cdot \frac{\partial(L+D X)_{p q}}{\partial X_{i i}} \\
& =-\left[(L+D X)^{-\mathrm{T}}(L+D X)^{-\mathrm{T}}\right]_{p q} \cdot \frac{\partial(L+D X)_{p q}}{\partial X_{i i}} \\
& =-\left[(L+D X)^{-\mathrm{T}}(L+D X)^{-\mathrm{T}}\right]_{p q} \cdot \frac{\partial(D X)_{p q}}{\partial X_{i i}} \\
& =-\left[(L+D X)^{-\mathrm{T}}(L+D X)^{-\mathrm{T}}\right]_{p q} \cdot \frac{\partial\left(D_{p m} \cdot X_{m q}\right)}{\partial X_{i i}} \\
& =-\left[\left[(L+D X)^{-\mathrm{T}}(L+D X)^{-\mathrm{T}}\right]_{p q} \cdot D_{p m} \cdot \delta_{m i} \cdot \delta_{q i}\right. \\
& =-\left[(L+D X)^{-\mathrm{T}}(L+D X)^{-\mathrm{T}}\right]_{p q} \cdot D_{p i} \cdot \delta_{q i} \\
& =-D_{i p}^{\mathrm{T}} \cdot\left[(L+D X)^{-\mathrm{T}}(L+D X)^{-\mathrm{T}}\right]_{p q} \cdot \delta_{q i} \\
& =-\left[D^{\mathrm{T}}(L+D X)^{-\mathrm{T}}(L+D X)^{-\mathrm{T}}\right]_{i i}
\end{aligned}
$$

where $p, q, m, i$ are indexes of elements in the matrix. Thus the Lemma is proven. $\square$

Definition 1. An angle $0 \leq \theta \leq \pi$ between two matrices $A$ and $B$ with the same dimension is defined as

$$
\theta=\arccos \left(\frac{\operatorname{tr}\left(A^{\mathrm{T}} B\right)}{\|A\|_{F}\|B\|_{F}}\right)
$$

where $\|\cdot\|_{F}$ represents the Frobenius norm of the matrix. The definition is reduced to an angle between two vectors when $A$ and $B$ become vectors.

Definition 2. For a matrix $A \in R^{N \times N}$, define $\{\cdot\}$ denote a vector form of an argument matrix constructed by stacking its column, and $\varrho(\cdot)$ as a 
column-wise operator which reshapes a dimensional vector back to a matrix. Thus, we have

$$
\varrho(\{A\})=A
$$

A. Revisited PGM (RPGM) for solving the optimization model (13)

Now we present a revisited projected gradient method (RPGM) to solve the optimization model (13). The main idea of the RPGM is to obtain the derivative of function $J(X)$ and $N(X)$ with respect to matrix $X$ respectively, and then project $\frac{\partial J(X)}{\partial X}$ onto a surface perpendicular to $\frac{\partial N(X)}{\partial X}$. The process is repeated until the iterative converges to the solution. The gradient of function $N(X)$ can be obtained as:

$$
\frac{\partial N(X)}{\partial X}=I
$$

Definition 3. Define a projection operator as

$$
P_{\frac{\partial N(X)}{\partial X}}(\nu)=\left(I-\left\{\frac{\partial N(X)}{\partial X}\right\}\left\{\frac{\partial N(X)}{\partial X}\right\}^{\dagger}\right) \nu
$$

where $\nu \in R^{N^{2} \times 1}$ is an arbitrary vector and $\dagger$ is the Moore Penrose pseudo inverse of a matrix.

In $[28,33]$, it has been shown that $P_{\frac{\partial N(X)}{\partial X}}(\nu)=\left(I-\left\{\frac{\partial N(X)}{\partial X}\right\}\left\{\frac{\partial N(X)}{\partial X}\right\}^{\dagger}\right) \nu$ was indeed a projection operator which projects $\nu$ onto the surface perpendicular to $\frac{\partial N(X)}{\partial X}$. The RPGM is presented as follows:

(1) Step 1. Set $k=0$ and initialize $X$ as a random matrix $X_{0}$ with a dimension $N \times N$ under the contraint $\operatorname{tr}\left(X_{0}\right)=N_{l}$.

(2) Step 2. At the iteration $k$, calculate the gradients of $\frac{\partial N(X)}{\partial X}$ and $\frac{\partial J(X)}{\partial X}$, respectively. Then calculate the projection

$$
\left\{\widetilde{\frac{\partial J(X)}{\partial X}}\right\}=\left(I_{N^{2}}-\left\{I_{N}\right\}\left\{I_{N}\right\}^{\dagger}\right)\left\{\frac{\partial J(X)}{\partial X}\right\}
$$


(3) Step 3. Update $X_{k}$ by projected gradient descent iteration $X_{k+1}=$ $X_{k}-\Delta X_{k}$ where

$$
\Delta X_{k}=-\eta \cdot \varrho\left(\left\{\frac{\widetilde{\partial J(X)}}{\partial X}\right\}\right)
$$

at $X=X_{k}$. Here $\eta$ is a step length, and $\varrho(\cdot)$ is a column-wise operator which reshapes a $N^{2} \times 1$ dimensional vector $\left\{\frac{\widetilde{\partial J(X)}}{\partial X}\right\}$ back to a $N \times N$ matrix $\frac{\widetilde{\partial J(X)}}{\partial X}$.

(4) Step 4. Calculate the angle $\cos \left(\theta_{k}\right)$ between $\nabla J\left(X_{k}\right)$ and $\Delta X_{k}$ based on Definition 1:

$$
\cos \left(\theta_{k}\right)=\left(\frac{\operatorname{tr}\left(\left[\nabla J\left(X_{k}\right)\right]^{T} \Delta X_{k}\right)}{\left\|\nabla J\left(X_{k}\right)\right\|_{F}\left\|\Delta X_{k}\right\|_{F}}\right)
$$

where $\nabla J\left(X_{k}\right)=\frac{\partial J(X)}{\partial X}$.

(5) Step 5. If $\left|\cos \left(\theta_{k}\right)\right|<\varepsilon$, then stop and let $X^{*}=X_{k}$; otherwise, update $k=k+1$ and go to Step 2. Here $\varepsilon$ is a positive small constant that is to set a termination criteria of the iteration process. The solution for the matrix $X_{k}$ obtained by RPGM is denoted as $X^{*}$.

Note that in RPGM, each element of $X_{i i}^{*}$ can be real value, and the absolute value of $X_{i i}^{*}$, denoted as $\left|X_{i i}^{*}\right|$ which actually evaluates how important node $i$ is to be selected as a leader. This implies that each node can be a leader in this case. Such a situation makes the above RPGM not realizable in practical applications since it may be physically impossible to select too many nodes as leaders. An interesting yet important issue is how to fix the number of leaders. To address this issue, we define an importance index

$$
r=\frac{\left[r_{1} \ldots r_{i} \ldots r_{N}\right]}{\max \left(r_{1}, \ldots, r_{i}, \ldots, r_{N}\right)}
$$

where $r_{i}=\left|X_{i i}^{*}\right|$ for all nodes $i=1, \ldots, N$. Clearly, we have $\max \{r\}=$ 1. It is seen that $r_{i}$ can be used to measure the importance of node $i$ to be a leader. So it is natural to select the nodes corresponding to the $M$ largest importance indexes as leaders. This method is termed as "projected gradient method extension" (PGME). However, such techniques may suffer from large performance penalty in real-life networks due to the diversity of 
different networks. In the following paragraph we shall develop a probabilistic projected gradient method (PPGM) to address this issue. In PPGM, the matrix variable $X$ denoted as $X^{b}$, becomes a Boolean variable and has a fixed trace.

\section{B. PPGM for solving the optimization model (12)}

The main idea of PPGM is that we apply a probabilistic projection operator $\mathcal{P}(\cdot)$ between the two spaces $X \in R^{N \times N}$ and $X^{b} \in\{0,1\}^{N \times N}$, and $X^{b}$ is iteratively searched in a Boolean matrix space $\{0,1\}^{\mathrm{N} \times \mathrm{N}}$. The process of PPGM is summarized as follows:

1) Step 1, Set $k=0$ and initialize $X$ as a random matrix $X_{0}$ with a dimension $N \times N$. Denote that $J_{0}^{\text {valid }}=J\left(X_{0}^{b}\right), X_{\text {best }}^{b}=X_{0}^{b}$ and $J_{b e s t}=J\left(X_{0}^{b}\right)$.

2) Step 2, (Probabilistic projection). Define a projection operator $\mathcal{P}(\cdot)$ and apply it to $X_{k}$, i.e., $X_{k}^{b}=\mathcal{P}\left(X_{k}\right)$, such that $X_{k}^{b}$ is a Boolean matrix satisfying the constraint $\operatorname{tr}\left(X_{k}^{b}\right)=N_{l}$. The detailed of the projection process is illustrated as follows:

a) Obtain the importance index vector $r=\frac{\left[r_{1} \ldots r_{i} \ldots r_{N}\right]}{\max \left(r_{1}, \ldots, r_{i}, \ldots, r_{N}\right)}$ defined in (24).

b) Let the node set corresponding to the $N_{l}+m_{0}\left(m_{0} \geq 1\right)$ largest maximum values of $r_{i}$ be a candidate set, denoted as $I_{\text {candidate, and }}$ the node set that shall be removed from the $I_{\text {candidate }}$ is denoted as $I_{\text {removed }}$. The number of nodes in $I_{\text {candidate }}$ is $N_{\text {candidate, and }}$ $N_{\text {removed }}$ has the same meaning for $I_{\text {removed }}$. At the beginning, we have:

$I_{\text {candidate }}=\left\{\right.$ nodes having the $N_{l}+m_{0}$ largest values of importance index $\}$

$N_{\text {candidate }}=N_{l}+m_{0}$

$I_{\text {removed }}=\{\phi\}$

$N_{\text {removed }}=0$

c) When $N_{\text {removed }}=m_{0}$ which means that $m_{0}$ nodes have been removed and the size of the $I_{\text {candidate }}$ becomes $N_{l}$, stop the probabilistic projection process and go to Step 3; otherwise, continue. 
d) Update the importance index vector for the nodes belongs to the candidate set:

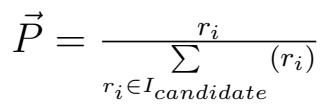

Then, $1-\vec{P}$ represents the probability distribution that nodes can be removed from $I_{\text {candidate }}$ to $I_{\text {removed }}$.

e) Sampling the probability vector $1-\vec{P}$ based on Monte Carlo method and obtain $1-\vec{P}=\left[\widetilde{p}_{1}, \ldots, \widetilde{p}_{i}, \ldots, \widetilde{p}_{N_{\text {candidate }}}\right]$. A node corresponding to the minimum $\widetilde{p}_{i}$ with $i=n_{\text {removed }}$ is chosen to be removed from $I_{\text {candidate }}$ to $I_{\text {removed }}$, we have:

$I_{\text {candidate }} \longleftarrow I_{\text {candidate }}-n_{\text {removed }}$

$N_{\text {candidate }} \longleftarrow N_{\text {candidate }}-1$

$I_{\text {removed }} \longleftarrow I_{\text {removed }}+n_{\text {removed }}$

$N_{\text {removed }} \longleftarrow N_{\text {removed }}+1$

f) go to $c$ ).

3) Step 3. If $J\left(X_{k}^{b}\right)<J_{b e s t}$, then

$$
\begin{aligned}
& J_{k}^{\text {valid }} \longleftarrow J\left(X_{k}^{b}\right) \\
& X_{\text {best }}^{b} \longleftarrow X_{k}^{b} \\
& J_{\text {best }} \longleftarrow J\left(X_{k}^{b}\right)
\end{aligned}
$$

and

$$
X_{k+1}=X_{k}^{b}-\Delta X_{k}
$$

where $\Delta X_{k}=-\eta \cdot \varrho\left(\left\{\frac{\widetilde{\partial J(X)}}{\partial X}\right\}\right), \eta$ is a step length, $\left\{\frac{\widetilde{\partial J(X)}}{\partial X}\right\}=$ $P_{\frac{\partial N(X)}{\partial X}}\left\{\frac{\partial J(X)}{\partial X}\right\}$ and $\varrho(\cdot)$ is a column-wise operator which reshapes a $N \cdot N$ dimensional vector $\left\{\widetilde{\frac{\partial J(X)}{\partial X}}\right\}$ back to a $N \times N$ matrix $\frac{\widetilde{\partial J(X)}}{\partial X}$. else

$$
\begin{aligned}
& J_{k}^{\text {valid }} \longleftarrow J_{k-1}^{\text {valid }} \\
& X_{k+1}=X_{k}-\Delta X_{k}
\end{aligned}
$$

end

4) Step 4. Update $k \longleftarrow k+1$. If $k<K^{*}$, go to Step 3; otherwise, stop.

Note that allowing $N_{l}+m_{0}\left(m_{0} \geq 1\right)$ and next removing $m_{0}$ nodes from the candidate set is important. Such a simple technique helps to reduce the variance of the deviation from consensus in stochastically forced networks. 
Through the PPGM algorithm, we obtain the $X_{\text {best }}^{b}$, which can be easily constructed: if node $i$ is a leader, we set $\left(X_{\text {best }}^{b}\right)_{i i}=1$; otherwise, $\left(X_{\text {best }}^{b}\right)_{i i}=0$.

\section{Convergence Analysis}

Before showing the convergence of our proposed iterative algorithms, we introduce following lemmas.

Lemma 5. For the matrix $A$ with $N \times M$, and the matrix $B$ with $N \times M$, note that the $\{A\}$ and $\{B\}$ are the vector form of both the matrix $A$ and matrix $B$, respectively. Then we have:

$$
\operatorname{tr}\left(A^{T} B\right)=\{A\}^{T}\{B\}
$$

Proof. We assume

$$
\begin{aligned}
\mathbf{A} & =\left(\begin{array}{cccc}
a_{11} & a_{12} & \ldots & a_{1 M} \\
a_{21} & a_{22} & \ldots & a_{2 M} \\
\vdots & \vdots & \ddots & \vdots \\
a_{N 1} & a_{N 2} & \ldots & a_{N M}
\end{array}\right) \\
\mathbf{B} & =\left(\begin{array}{cccc}
b_{11} & b_{12} & \ldots & b_{1 M} \\
b_{21} & b_{22} & \ldots & b_{2 M} \\
\vdots & \vdots & \ddots & \vdots \\
b_{N 1} & b_{N 2} & \ldots & b_{N M}
\end{array}\right)
\end{aligned}
$$

Then $\operatorname{tr}\left(A^{T} B\right)=\sum_{j=1}^{M} \sum_{i=1}^{N} a_{i j} b_{i j}=\{A\}^{T}\{B\}$

Lemma 6. For all $X \in$ dom $g$ (the domain of $g$ ), any $Y \in R^{k \times l}$, and some open interal of $t \in R$, we have

$$
\begin{aligned}
g(X+t Y) & =g(X)+t \cdot \vec{d}(X)+o\left(t^{2}\right) \\
& =g(X)+t \cdot \operatorname{tr}\left(\nabla g(X)^{T} Y\right)+o\left(t^{2}\right)
\end{aligned}
$$

which is the first-order Taylor series expansion about $X$. It has been proven in [34]. 
Theorem 1. The sequence $X_{k}$ generated in (22) converges to a local optimal point of $J(X)$ satisfying first-order optimality condition for a given sufficient small $\eta$.

Proof. We define

$$
J\left(X_{k}\right)=\operatorname{trace}\left((L+D X)^{-1}\right)
$$

where $k$ denotes the $k$-th iteration. To prove the convergence, we have to show that

$$
J\left(X_{k+1}\right)-J\left(X_{k}\right) \leq 0 \text {, and } \quad J\left(X_{k}\right) \geq 0
$$

Because $L+D X$ is a position semidefinite matrix, then $J\left(X_{k}\right) \geq 0$.

From the equation (22), we can obtain

$$
\begin{aligned}
\operatorname{tr}\left(X_{k+1}\right) & =\operatorname{tr}\left(X_{k}-\eta \cdot \varrho\left(P_{\frac{\partial N(X)}{\partial X}}\left\{\nabla J\left(X_{k}\right)\right\}\right)\right) \\
& =\operatorname{tr}\left(X_{k}\right)-\eta \cdot \operatorname{tr}\left(\varrho\left(P_{\frac{\partial N(X)}{\partial X}}\left\{\nabla J\left(X_{k}\right)\right\}\right)\right) \\
& =\operatorname{tr}\left(X_{k}\right)-\eta \cdot\left\{\varrho\left(P_{\frac{\partial N(X)}{\partial X}}\left\{\nabla J\left(X_{k}\right)\right\}\right)\right\}^{T}\left\{I_{N}\right\}
\end{aligned}
$$

From the Definition 2, we can obtain

$$
\begin{aligned}
& \operatorname{tr}\left(X_{k}\right)-\eta \cdot\left\{\varrho\left(P_{\frac{\partial N(X)}{\partial X}}\left\{\nabla J\left(X_{k}\right)\right\}\right)\right\}^{T}\left\{I_{N}\right\} \\
& =\operatorname{tr}\left(X_{k}\right)-\eta \cdot\left(P_{\frac{\partial N(X)}{\partial X}}\left\{\nabla J\left(X_{k}\right)\right\}\right)^{T}\left\{I_{N}\right\} \\
& =\operatorname{tr}\left(X_{k}\right)-\eta \cdot\left\{\nabla J\left(X_{k}\right)\right\}^{T} P_{\frac{\partial N(X)}{\partial X}}\left\{I_{N}\right\} \\
& =\operatorname{tr}\left(X_{k}\right)-\left\{\nabla J\left(X_{k}\right)\right\}^{T}\left(I_{N^{2}}-\left\{I_{N}\right\}\left\{I_{N}\right\}^{\dagger}\right)\left\{I_{N}\right\} \\
& =\operatorname{tr}\left(X_{k}\right)
\end{aligned}
$$

where $I_{N}$ is an identity matrix with dimensional $N \times N$ and $I_{N^{2}}$ is an identity matrix with dimensional $N^{2} \times N^{2}$. Then the $\operatorname{tr}\left(X_{k+1}\right)=\operatorname{tr}\left(X_{k}\right)$ is achieved. Thus from equation (22), we have

$$
X_{k+1}=X_{k}-\eta \cdot \varrho\left(P_{\frac{\partial N(X)}{\partial X}}\left\{\nabla J\left(X_{k}\right)\right\}\right)
$$

Then from the Lemma 5 and Lemma 6, we obtain

$$
\begin{aligned}
J\left(X_{k+1}\right) & =J\left(X_{k}-\eta \cdot \varrho\left(P_{\frac{\partial N(X)}{\partial X}}\left\{\nabla J\left(X_{k}\right)\right\}\right)\right) \\
& =J\left(X_{k}\right)-\eta \cdot \operatorname{tr}\left(\nabla J\left(X_{k}\right)^{T} \varrho\left(P_{\frac{\partial N(X)}{\partial X}}\left\{\nabla J\left(X_{k}\right)\right\}\right)\right) \\
& =J\left(X_{k}\right)-\eta \cdot\left\{\nabla J\left(X_{k}\right)^{T}\right\}\left\{\varrho\left(P_{\frac{\partial N(X)}{\partial X}}\left\{\nabla J\left(X_{k}\right)\right\}\right)\right\} \\
& =J\left(X_{k}\right)-\eta \cdot\left\{\nabla J\left(X_{k}\right)^{T}\right\} P_{\frac{\partial N(X)}{\partial X}}\left\{\nabla J\left(X_{k}\right)\right\}
\end{aligned}
$$


Thus

$$
J\left(X_{k+1}\right)-J\left(X_{k}\right)=-\eta \cdot\left\{\nabla J\left(X_{k}\right)^{T}\right\} P_{\frac{\partial N(X)}{\partial X}}\left\{\nabla J\left(X_{k}\right)\right\}
$$

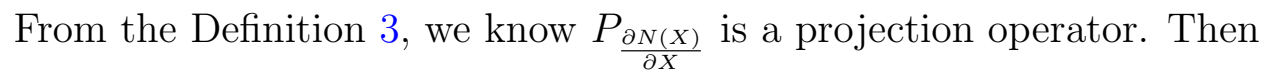

$$
\begin{aligned}
& \left\{\nabla J\left(X_{k}\right)^{T}\right\} P_{\frac{\partial N(X)}{\partial X}}\left\{\nabla J\left(X_{k}\right)\right\} \\
& =\left\{\nabla J\left(X_{k}\right)^{T}\right\} P_{\frac{\partial N(X)}{\partial X}}^{T} P_{\frac{\partial N(X)}{\partial X}}\left\{\nabla J\left(X_{k}\right)\right\} \\
& =\left(P_{\frac{\partial N(X)}{\partial X}}\left\{\nabla J\left(X_{k}\right)\right\}\right)^{T} P_{\frac{\partial N(X)}{\partial X}}\left\{\nabla J\left(X_{k}\right)\right\}
\end{aligned}
$$

Assume that $\lambda_{i}\left(i=1,2, \cdots, N^{2}\right)$ are the eigenvalues of $\frac{P_{\frac{\partial N(X)}{\partial X}}}{\partial}\left\{\nabla\left(X_{k}\right)\right\}$, which implies that

$$
\begin{aligned}
& \left(P_{\frac{\partial N(X)}{\partial X}}\left\{\nabla J\left(X_{k}\right)\right\}\right)^{T} P_{\frac{\partial N(X)}{\partial X}}\left\{\nabla J\left(X_{k}\right)\right\} \\
& =\eta\left(\sum_{i} \lambda_{i}^{2}\right) \\
& \geq 0
\end{aligned}
$$

As $\eta$ is a sufficiently small positive number, we obtain that

$$
\begin{aligned}
& \lim _{\eta \rightarrow 0^{+}}\left(J\left(X_{k+1}\right)-J\left(X_{k}\right)\right) \\
& =\lim _{\eta \rightarrow 0^{+}}\left(-\eta\left\{\nabla J\left(X_{k}\right)^{T}\right\} P_{\frac{\partial N(X)}{\partial X}}\left\{\nabla J\left(X_{k}\right)\right\}\right) \\
& \leq 0
\end{aligned}
$$

From above, when $\eta$ is small enough, the iteration is convergent. The iteration stops when the $\left.\frac{P_{\frac{\partial N(X)}{\partial X}}}{\partial J} J\left(X_{k}\right)\right\}=0$.

Theorem 2. The iterative algorithm of RPGM converges linearly to $J\left(X^{*}\right)$.

Proof. From equation (32)-(34), we know that

$$
\begin{aligned}
& J\left(X_{k}\right)-J\left(X_{k+1}\right) \\
& =\eta\left\{\nabla J\left(X_{k}\right)^{T}\right\} P_{\frac{\partial N(X)}{\partial X}}\left\{\nabla J\left(X_{k}\right)\right\} \\
& =\eta\left(\sum_{i} \lambda_{i}^{2}\right)
\end{aligned}
$$

Subtracting $J\left(X^{*}\right)$ on both sides, we obtain that

$$
\begin{aligned}
& J\left(X_{k}\right)-J\left(X_{k+1}\right) \\
& =J\left(X_{k}\right)-J\left(X^{*}\right)-\left(J\left(X_{k+1}\right)-J\left(X^{*}\right)\right) \\
& =\eta\left(\sum_{i} \lambda_{i}^{2}\right)
\end{aligned}
$$


then

$$
\begin{aligned}
& J\left(X_{k}\right)-J\left(X^{*}\right) \\
& =J\left(X_{k+1}\right)-J\left(X^{*}\right)+\eta\left(\sum_{i} \lambda_{i}^{2}\right)
\end{aligned}
$$

both sides of equation (38) divided by $J\left(X_{k}\right)-J\left(X^{*}\right)$, then

$$
1=\frac{J\left(X_{k+1}\right)-J\left(X^{*}\right)}{J\left(X_{k}\right)-J\left(X^{*}\right)}+\frac{\eta\left(\sum_{i} \lambda_{i}^{2}\right)}{J\left(X_{k}\right)-J\left(X^{*}\right)}
$$

then

$$
\frac{J\left(X_{k+1}\right)-J\left(X^{*}\right)}{J\left(X_{k}\right)-J\left(X^{*}\right)}=1-\frac{\eta\left(\sum_{i} \lambda_{i}^{2}\right)}{J\left(X_{k}\right)-J\left(X^{*}\right)}
$$

Because $J\left(X_{k}\right)-J\left(X^{*}\right) \geq 0$ and $\eta\left(\sum_{i} \lambda_{i}^{2}\right) \geq 0$, so $\frac{\eta\left(\sum_{i} \lambda_{i}^{2}\right)}{J\left(X_{k}\right)-J\left(X^{*}\right)} \geq 0$. Thus $\frac{J\left(X_{k+1}\right)-J\left(X^{*}\right)}{J\left(X_{k}\right)-J\left(X^{*}\right)} \leq 1$. This implies that

$$
\frac{J\left(X_{k+1}\right)-J\left(X^{*}\right)}{J\left(X_{k}\right)-J\left(X^{*}\right)}=\beta_{k}
$$

Then, we obtain $\lim _{k \rightarrow \infty} \sup \left(\beta_{k}\right)=1$, so it proves that the RPGM iterative algorithm has at least linear convergence.

Remark 1. In implementations, the computation time of RPGM depends on the convergence rate, the computational complexity as well as memory complexity in each iteration. Theorem 2 shows that the convergence rate of RPGM is at least linear. In each iteration, computation complexity mainly depends on how to calculate the $\left(I_{N^{2}}-\left\{I_{N}\right\}\left\{I_{N}\right\}^{\dagger}\right)\left\{\frac{\partial J(X)}{\partial X}\right\}$ which involves matrix multiplication and matrix inverse (do the matrix inverse of a $N \times N$ dimensional matrix $(L+D X))$. On the one side, $\left(I_{N^{2}}-\left\{I_{N}\right\}\left\{I_{N}\right\}^{\dagger}\right)$ is a sparse matrix and it has only $N$ none zero elements. Therefore the computational complexity of calculating $\left(I_{N^{2}}-\left\{I_{N}\right\}\left\{I_{N}\right\}^{\dagger}\right)$ is $o\left(N^{2}\right)$ with memory complexity being $o(N)$. On the other side, the computational complexity of calculating $\frac{\partial J(X)}{\partial X}$ is also $o\left(N^{2}\right)$ with memory complexity being $o\left(N^{2}\right)$ since the rank one update of Moore-penrose inverse of $L+D X$ could be applied [35]. Thus, both the computational complexity and the memory complexity of RPGM at each iteration are $o\left(N^{2}\right)$. Due to the similar iteration process, such a conclusion is still valid for PPGM. However, the probabilistic projection is involved at each step of PPGM, which leads to a slightly higher computational cost compared with RPGM. 
Remark 2. Theorem 1 shows that the sequence $X_{k}$ converges to a local minimum point. Recall that the optimization model in (13) is convex. Therefore, a local minimum point of (13) is also a global minimum point. On the other side, for the original non-convex optimization model (12) solved by PPGM, its convergence in this case is vivid, since we always have $J\left(X_{k}^{\text {valid }}\right) \geq$

$J\left(X_{k+1}^{\text {valid }}\right) \geq 0$ as seen in the step 3 . In the case studies section below, it will be shown that PPGM steadily approaches the global minimum point obtained by RPGM in various synthetic and real-life applications.

\section{Case Studies}

We apply RPGM and PPGM to the leader selection problem in a small random network, $19 \times 19$ Go network and some real-life networks. We further compare these two methods with the state-of-art techniques including greedy algorithm (GA) [21] and PGM-extension (PGME) [28]. We assume connected and undirected networks.

Case 1: (a small random network.) We consider the selection of noisecorrupted leaders in a network with 100 nodes randomly distributed over a unit square area. A pair of nodes can communicate with each other if their distance is no greater than 0.2 units. This scenario arises in sensor networks with prescribed omnidirectional sensing range [1,36]. Fig.1 shows the performances of RPGM, PPGM, GA and PGME for the leader selection in this network. It is observed that a) the lower bound cost can be obtained in this random network by RPGM; b) the cost obtained by both PPGM and GA are almost the same; c) the cost of PGME fluctuates sometimes especially when $N_{l}$ is relatively small; d) the cost decreases significantly when number of $N_{l}$ increases, in other words, having more leaders can steer followers effectively and improve the performance of the network. It is well known that installing more leaders may induce other costs. In real-life applications, there is a trade-off between the number of leaders and the cost.

Case 2: (Go network.) We apply the PPGM and GA to identify $N_{l}$ leaders in $19 \times 19$ Go network with 361 nodes [37]. Simulation results reveal some exciting phenomena as shown in Fig.2. By applying PPGM, when $N_{l}=4$, nodes $(2,2),(18,18)$ and $(2,18),(18,2)$ are selected, which exhibits symmetry with respect to the center of the Go network. For $N_{l}=8$, nodes $(2,2),(2,12),(2,18),(8,3)$ and $(18,18),(18,11),(18,2),(11,17)$ are selected, as well as $N_{l}=16$. Most of the selected leaders locate near the boundary 
Table 1: Comparison results in real-life networks

\begin{tabular}{|c|c|c|c|c|c|c|c|c|c|}
\hline Date Set & Networks & Nodes & $N_{l}$ & $X_{0}$ & RPGM & $X_{0}^{\mathrm{b}}$ & PPGM & GA & PGME \\
\hline \multirow[t]{3}{*}{ Electronic Circuit } & Circuit-s208 & 122 & 50 & 44.7611 & 40.6565 & 56.8606 & 47.0131 & 46.9531 & 48.3803 \\
\hline & Circuit-s 420 & 252 & 80 & 100.4713 & 91.8012 & 127.2253 & 107.9443 & 106.8015 & 114.7599 \\
\hline & Circuit-s838 & 512 & 150 & 211.3537 & 190.4649 & 274.4437 & 224.6036 & 220.9302 & 237.5845 \\
\hline \multirow[t]{2}{*}{ Food Web } & CrystalD & 24 & 10 & 3.5089 & 3.2920 & 4.0702 & 3.7649 & 3.7649 & 3.9974 \\
\hline & Florida & 128 & 50 & 4.0443 & 3.5668 & 4.5981 & 3.9179 & 3.9168 & 3.9207 \\
\hline \multirow[t]{2}{*}{ Social } & Con-freq-rev & 46 & 15 & 1.5079 & 1.2817 & 1.6011 & 1.4579 & 1.4577 & 1.5112 \\
\hline & Con-quality-rev & 46 & 15 & 1.9949 & 1.4308 & 2.0782 & 1.7399 & 1.7396 & 1.8157 \\
\hline Neural & Celegans & 297 & 100 & 32.7282 & 25.8979 & 37.0120 & 28.6904 & 28.6783 & 28.7333 \\
\hline Airport & Us & 500 & 150 & 78.5765 & 64.5249 & 91.5196 & 70.1180 & 69.7765 & 71.5907 \\
\hline
\end{tabular}

of the grid. Furthermore, we can see from Fig.2 that the leaders selected by PPGM are similar to those selected by GA. The comparison results are shown in Fig.3. The conclusions claimed in Case 1 can also be obtained in this case.

Case 3: (real-life networks.) We also compare the performance of RPGM, PPGM, GA and PGME in some real-life networks [38-47]. The results are shown in Table I, where the initial and binary initial value of $X$ is denoted as $X_{0}$ and $X_{0}^{b}$, respectively. Table I shows that the RPGM and the other three algorithms reduce the cost efficiently comparing to the cost with $X_{0}$

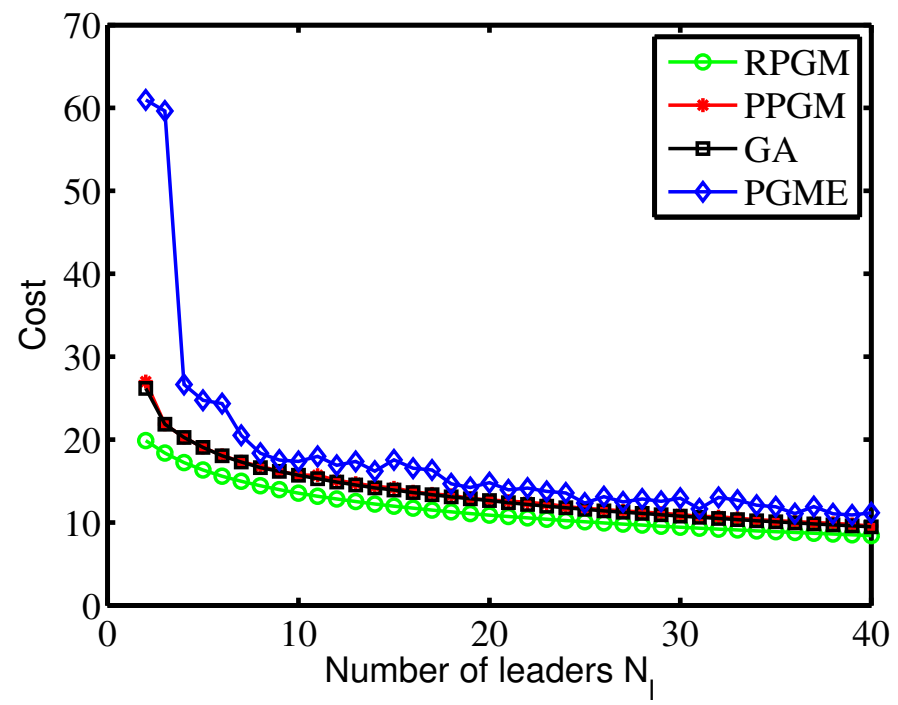

Figure 1: The comparison results of RPGM, PPGM, GA and PGME in a random network. 


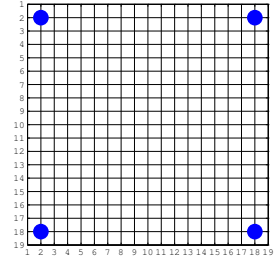

(a)

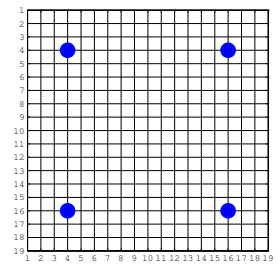

(d)

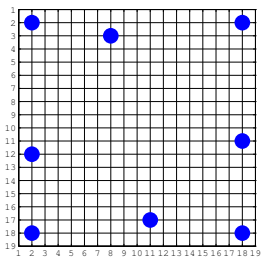

(b)

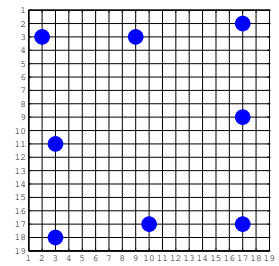

(e)

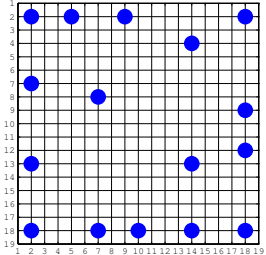

(c)

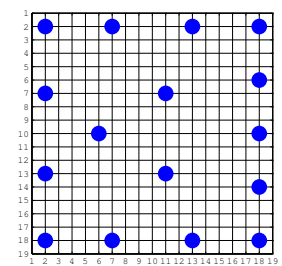

(f)

Figure 2: Selection of leaders for a Go network example using PPGM in (a), (b) and (c) and using GA in (d), (e) and (f). (a) PPGM: the selected leaders $N_{l}=4$; (b) PPGM: the selected leaders $N_{l}=8$; (c) PPGM: the selected leaders $N_{l}=16$; (e) GA: the selected leaders $N_{l}=4$; (f) GA: the selected leaders $N_{l}=8$; (f) GA: the selected leaders $N_{l}=16$.

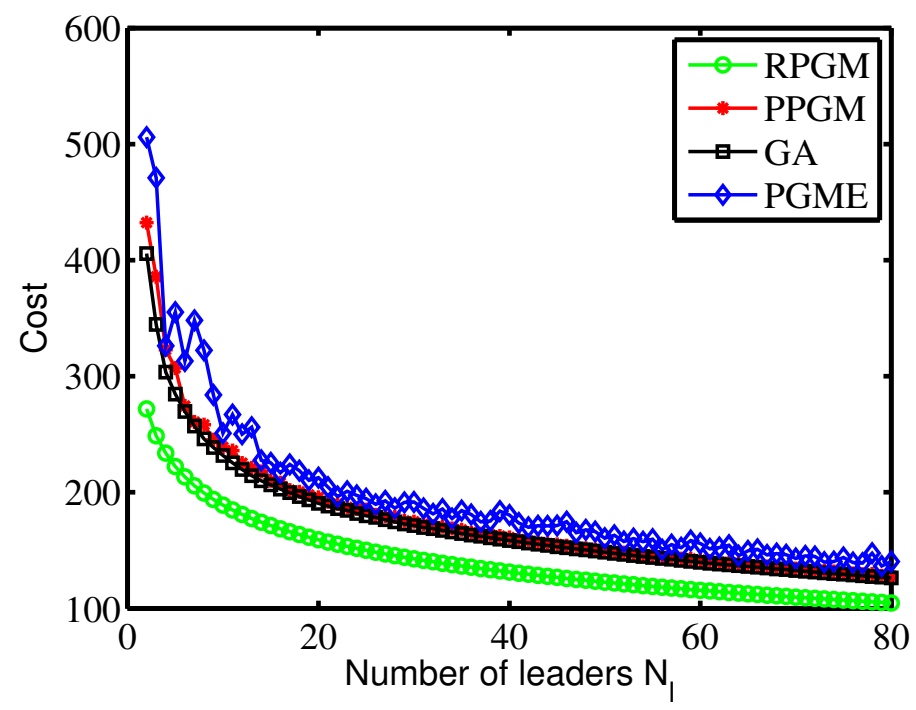

Figure 3: The comparison results of RPGM, PPGM, GA and PGME in a Go network 
and $X_{0}^{b}$. Furthermore, it verifies that a) the developed PPGM has a comparable performance with GA; b) PPGM steadily approaches the RPGM and outperforms PGME. Fig.4 illustrates a small real-life network topology with the selected leaders by PPGM.

In order to show the time cost of PPGM and RPGM, we established different sizes $(N=10,20,30,40,50,60,70,80,90,100,150,200,250,300,350$, $400,450,500$ nodes) of random networks by predefining $0.2 N$ nodes as leaders. The comparisons of the time cost of PPGM and RPGM in 300 iteration steps are shown in Fig.5. Clearly, PPGM has a slightly higher computational cost compared with RPGM which is consistent with the Remark 1 where the probabilistic projection is involved at each step of PPGM .

At last, the illustration of the convergence property of RPGM is shown in Fig.6(a) where $\cos \left(\theta_{k}\right)$ is gradually converges to 0 , and $\cos \left(\theta_{k}\right)<\varepsilon$ can be set as a termination criteria for the iteration process. The convergence of PPGM is vivid and the cost is gradually reduced as show in Fig.6(b). They suggest the effectiveness of the employing of the RPGM and PPGM algorithms.

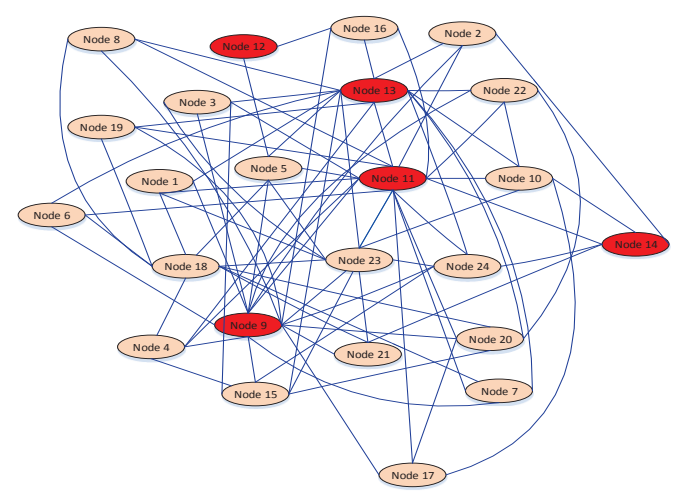

Figure 4: A small real-life network topology [38] with the selected leaders by PPGM. The selected leader nodes are marked in red. 


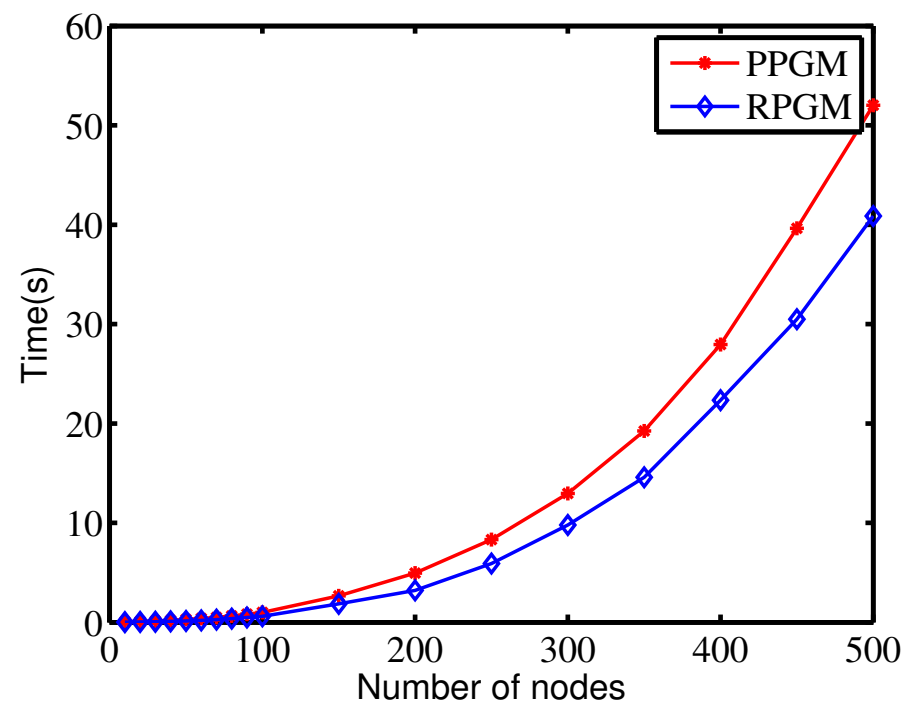

Figure 5: The time cost of PPGM and RPGM with different sizes of random networks.

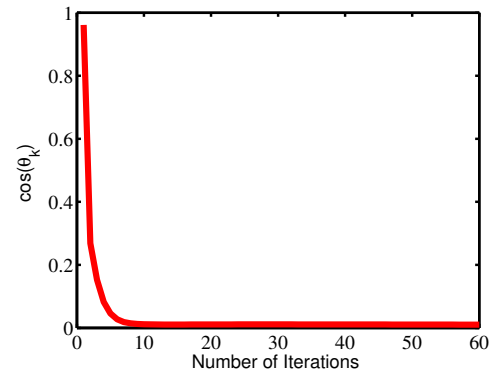

(a)

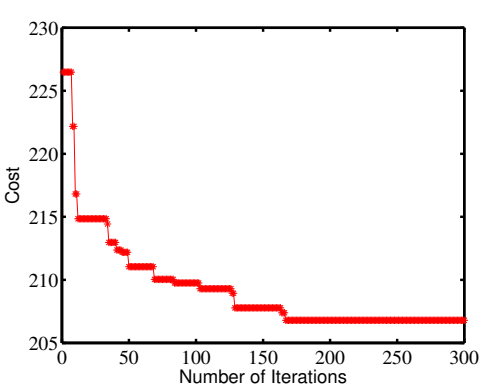

(b)

Figure 6: Iteration process. (a) the $\cos \left(\theta_{k}\right)$ obtained by RPGM converges to 0 asymptotically; (b) the cost obtained by PPGM is gradually reduced. 


\section{Conclusions and Discussions}

In this paper, leader selection problem in stochastically forced consensus networks is studied. A chain rule for matrix differentiation is introduced to obtain the gradient of the cost function involving matrix variables. Two optimization models are formulated which can be solved by two iterative algorithms named revisited projected gradient method (RPGM) and probabilistic projected gradient method (PPGM), respectively. The convergence property of both methods is established. Simulation results show satisfactory performance of the two iterative algorithms compared to greedy algorithm (GA) and PGM-extension (PGME). The proposed algorithms may bring new insights to various applications. Along this direction, many studies can be carried out in the further, e.g., to develop simpler heuristic algorithms for selecting the leader node set in extra-large networks.

Acknowledgement: The work was supported partially by National Science Foundation of China (61603209), and Beijing Natural Science Foundation (4164086), and National Basic Research Program of China (973 Program, Grant No. 2015CB057406), and Independent Research Plan of Tsinghua University (20151080467).

[1] M. Mesbahi, M. Egerstedt, Graph theoretic methods in multiagent networks, Princeton University Press, 2010.

[2] J. Cortes, Distributed algorithms for reaching consensus on general functions, Automatica 44 (3) (2008) 726-737.

[3] E. Mossel, G. Schoenebeck, Reaching consensus on social networks, innovations in Computer Science, Tsinghua University, Beijing, China, January (2010) 214-229.

[4] R. Hegselmann, U. Krause, Opinion dynamics and bounded confidence models, analysis and simulation, Journal of Artificial Societies and Social Simulation 5 (3) (2002) 1-33.

[5] M. H. Degroot, Reaching a consensus, Journal of the American Statistical Association 69 (345) (1974) 118-121. 
[6] B. Golub, M. O. Jackson, Naive learning in social networks and the wisdom of crowds, American Economic Journal: Microeconomics 2 (1) (2010) 112-149.

[7] J. A. Fax, R. M. Murray, Information flow and cooperative control of vehicle formations, IEEE Transactions on Automatic Control 49 (9) (2004) $1465-1476$.

[8] A. Jadbabaie, J. Lin, A. S. Morse, Coordination of groups of mobile autonomous agents using nearest neighbor rules, American Conference on Decision and Control 50 (11) (2003) 988-1001.

[9] P. Ogren, E. Fiorelli, N. E. Leonard, Cooperative control of mobile sensor networks: Adaptive gradient climbing in a distributed environment, IEEE Transactions on Automatic Control 49 (8) (2004) 1292-1302.

[10] P. A. Bliman, G. Ferrari-Trecate, Average consensus problems in networks of agents with delayed communications, Automatica 44 (8) (2008) 1985-1995.

[11] P. Barooah, J. P. Hespanha, Estimation on graphs from relative measurements, IEEE Control Systems 27 (4) (2007) 57-74.

[12] L. Ioannis, V. Glenn, Scalable robustness for consensus protocols with heterogeneous dynamics, Proceedings of the 16th IFAC world congress (2005) 185-190.

[13] J. Wang, Y. Tan, I. Mareels, Robustness analysis of leader-follower consensus, Journal of systems science and complexity 22 (2) (2009) 186-206.

[14] G. F. Young, L. Scardovi, N. E. Leonard, Robustness of noisy consensus dynamics with directed communication, in: American Control Conference, Marriott Waterfront, Baltimore, MD, USA, 2010, pp. 6312-6317.

[15] H. Fan, B. Liu, Y. Shen, W. Wang, Adaptive failure compensation control for uncertain systems with stochastic actuator failures, IEEE Transactions on Automatic Control 59 (3) (2014) 808-814.

[16] B. Bamieh, M. R. Jovanovin, P. Mitra, S. Patterson, Coherence in large-scale networks: Dimension-dependent limitations of local feedback, IEEE Transactions on Automatic Control 57 (9) (2011) 2235-2249. 
[17] D. Zelazo, M. Mesbahi, Edge agreement: Graph-theoretic performance bounds and passivity analysis, IEEE Transactions on Automatic Control 53 (6) (2011) 544-555.

[18] P. Barooah, J. P. Hespanha, Estimation from relative measurements: Electrical analogy and large graphs, IEEE Transactions on Signal Processing 56 (6) (2008) 2181-2193.

[19] L. Xiao, S. Boyd, S.-J. Kimb, Distributed average consensus with leastmean-square deviation, Journal of Parallel and Distributed Computing 67 (1) (2007) 33-46.

[20] F. Lin, M. Fardad, M. R. Jovanovic, Optimal control of vehicular formations with nearest neighbor interactions, IEEE Transactions on Automatic Control 57 (9) (2012) 2203-2218.

[21] F. Lin, M. Fardad, M. R. Jovanovic, Algorithms for leader selection in stochastically forced consensus networks, IEEE Transactions on Automatic Control 59 (7) (2014) 1789-1802.

[22] T. Borsche, S. A. Attia, On leader election in multi-agent control systems, in: Chinese Control and Decision Conference, 2010, pp. 102-107.

[23] S. Patterson, B. Bamieh, Leader selection for optimal network coherence, in: 49th IEEE Conference on Decision and Control, 2010, pp. 2692-2697.

[24] K. Fitch, N. E. Leonard, Information centrality and optimal leader selection in noisy networks, in: 52nd IEEE Conference on Decision and Control, 2013, pp. 7510-7515.

[25] A. Clark, L. Bushnell, R. Poovendran, A supermodular optimization framework for leader selection under link noise in linear multi-agent systems, IEEE Transactions on Automatic Control 59 (2) (2014) 283296.

[26] H. Kawashima, M. Egerstedt, Leader selection via the manipulability of leader-follower networks, in: American Control Conference (ACC), 2012, pp. 6053-6058. 
[27] G. Shi, K. C. Sou, H. Sandberg, K. H. Johansson, A graph-theoretic approach on optimizing informed-node selection in multi-agent tracking control, Physica D: Nonlinear Phenomena 267 (1) (2014) 104-111.

[28] G. Li, W. Hu, G. Xiao, L. Deng, P. Tang, J. Pei, L. Shi, Minimum-cost control of complex networks, New Journal of Physics 18 (1) (2016) 1-11.

[29] G. Li, P. Tang, C. Wen, Z. Meng, Boundary constraints for minimum cost control of directed networks, Submitted to IEEE Transactions on Cybernetics.

[30] R. Diestel, Graph Theory, Springer, Springer-Verlag Berlin Heidelberg, 2010.

[31] W. Ren, Y. Cao, Distributed Coordination of Multi-agent Networks, Communications and Control Engineering, 2010.

[32] K. B. Petersen, M. S. Pedersen, The Matrix Cookbook, 2012.

[33] G. Li, C. Wen, W. X. Zheng, Y. Chen, Identification of a class of nonlinear autoregressive models with exogenous inputs based on kernel machines, IEEE Transactions on Signal Processing 59 (5) (2011) 2146-2159.

[34] M. Dattorro, J. Dattorro, Convex Optimization and Euclidean Distance Geometry, Meboo Publishing USA, 2005.

[35] J. A. Fill, D. E. Fishkind, The moore penrose generalized inverse for sums of matrices, SIAM Journal on Matrix Analysis and Applications 21 (2) (2000) 629-635.

[36] S. Srirangarajan, A. H. Tewfik, Z.-Q. Luo, Distributed sensor network localization using socp relaxation, IEEE Transactions on Wireless Communications 7 (12) (2008) 4886-4895.

[37] H. Fan, C. Wen, Adaptive control of a class of 2-d discrete systems, IEEE Transactions on Circuits on Systems-I:Fundamental Theory and Applications 50 (1) (2003) 166-172.

[38] R. R. Christian, J. J. Luczkovich, Organizing and understanding a winter's seagrass foodweb network through effective trophic levels, Ecological Modelling 117 (1) (1999) 99-124. 
[39] R. Milo, S. Itzkovitz, N. Kashtan, et al., Superfamilies of evolved and designed networks, Science 303 (5663) (2004) 1538-1542.

[40] M. E. Monaco, R. E. Ulanowicz, Comparative ecosystem trophic structure of three u.s. mid-atlantic estuaries, Marine Ecology Progress 161 (8) (1997) 239-254.

[41] J. Almunia, G. Basterretxea, J. Aristegui, R. E. Ulanowicz, Benthicpelagic switching in a coastal subtropical lagoon, Estuarine, Coastal and Shelf Science 49 (3) (1999) 363-384.

[42] D. Baird, J. Luczkovich, R. R. Christian, Assessment of spatial and temporal variability in ecosystem attributes of the st marks national wildlife refuge, apalachee bay, florida, Estuarine, Coastal and Shelf Science 49 (3) (1998) 329-349.

[43] D. Baird, R. E. Ulanowicz, The seasonal dynamics of the chesapeake bay ecosystem, Ecological Monographs 59 (4) (1989) 329-364.

[44] R. S. Bur, Social contagion and innovation: Cohesion versus structural equivalence, American Journal of Sociology 92 (6) (1987) 1287-1335.

[45] M. P. Young, The organization of neural systems in the primate cerebral cortex, Proceedings of the Royal Society B Biological Sciences 252 (1333) (1993) 13-18.

[46] T. Opsahl, F. Agneessens, J. Skvoretzc, Node centrality in weighted networks: Generalizing degree and shortest paths, Social Networks 32 (3) (2010) 245-251.

[47] J. Marcelino, M. Kaiser, Critical paths in a metapopulation model of h1n1: Efficiently delaying influenza spreading through flight cancellation, Plos Currents 4. 